\title{
MODERN RAILWAYS: CONNECTING TRAIN CONTROL SYSTEMS WITH MOBILE AND SATCOM TELECOM NETWORKS
}

\author{
FRANCESCO RISPOLI \\ Hitachi Rail STS, Italy
}

\begin{abstract}
The growth of cellular and satellite telecom systems with the introduction of $5 \mathrm{G}$ and broadband mobile satellites are creating a global and pervasive telecom infrastructure offering connectivity services in any place in the world. Furthermore, 5G will provide millisecond latencies and network "slicing" capabilities that are ideal to realize bespoke virtual networks. A new generation of small satellites in low earth orbits are being launched promising a lower cost and a much higher throughput with latency below $100 \mathrm{~m} / \mathrm{s}$. The European Railways Train Management System (ERTMS) utilizes, since its conception in the early 1990s, GSM-R as the legacy telecom network linking trains with the Radio Block Center. This technology will have to be substituted as it approaches its end of life and cannot satisfy increasing demand of connectivity for new railway applications. UIC has defined the user needs of the Future Railway Mobile Communication System (FRMCS), one of the ERTMS game changers and the Shift2Rail - X2Rail-1 partners have agreed on adopting the ACS (Adaptable Communication System) as the architecture for the successor of the GSM-R. This paper addresses new railway needs with the goal of sharing cellular and satcom public telecom networks. An open multi-bearer system, coherent with ACS and FRMCS is evaluated as an alternative or complement to a dedicated network. A number of possible scenarios with different line lengths and operating trains demonstrate important cost reductions when bundling cellular and satellite bearers to achieve the QoS. Side benefits such as the possible reduction of new spectrum for the FRMCS applications, less emissions of $\mathrm{CO}_{2}$ and a faster time to operate are evaluated. Yet, the role of new high throughput satcom is underlined to satisfy the increasing demand of train connectivity and also to extend the coverage of the future $5 \mathrm{G}$ services in the railways ecosystem.
\end{abstract}

Keywords: ERTMS evolution, public telecom networks, satcom, $5 G$.

\section{INTRODUCTION}

All European countries are moving towards implementing ERTMS at different speed as pointed out by the report of the European Court of Auditors (ECA) [1]. In fact although European countries have agreed to deploy European Railways Train Management System (ERTMS) on their network the current low status of ERTMS deployment is probably explained by the perceived high investments and the lack of an individual business case. In this respect, one important cost element of the ERTMS is due to the deployment, operation and maintenance of the GSM-R network that coupled with ETCS, as constituents of ERTMS, provide an interoperable railway signalling and communication system for all the European railway networks operating in the $876-880 \mathrm{MHz}$ (train-to-ground) and $921-925 \mathrm{MHz}$ (ground-to-train) bands. Meanwhile, Future Railway Mobile Communication System (FRMCS) has been designated by UIC [2] as the successor of GSM-R, implying that both GSM-R and FRMCS will have to operate in parallel until a full migration to the FRMCS has been completed. The overall migration throughout Europe is expected to take place between 2022 and 2035 with scenarios depending on plans of each country to replace the current GSM-R infrastructure.

The Adaptable Communication System (ACS) is the selected new architecture based on the principle of bearer independence, overcoming the identification of a legacy technology 
such as the GSM in the early 1990s [3]. ACS will be using packet switching/IP technologies as LTE, Satellite, Wi-Fi to comply with the growing FRMCS user needs. In this evolving scenario the choice of the telecom bearer becomes a matter of economical convenience opening the way also to use cellular and satellite telecom services. In that case a servicebased model could be implemented as part of the ERTMS system delivery instead of building and operating a dedicated telecom network. The use of public networks, compatible with the ACS bearer-independent architecture, will bring a substantial reduction of costs during the operational life time of the ERTMS because the investments, licencing and operations are undertaken by cellular and satellite operators to keep their networks competitive with market needs. Furthermore is not hard to predict the impact of these public services as early adopter and promoter of future 5G connectivity for the ERTMS. Most satellite broadband providers are recognising the demand arising from the ERTMS evolution and the opportunity to provide a worldwide connectivity for the railways sector. Inmarsat, Thuraya and Iridium are delivering narrow-band connectivity at global level in the L-band, while Intelsat is using the $\mathrm{Ku}$-band to provide higher throughput with planar phased array antennas on trains. New highpower geostationary satellites under development for mobile applications in $\mathrm{Ku}$ and $\mathrm{Ka}$ bands will further improve the bitrate and capacity. Yet in perspective, low earth orbiting megaconstellation satellites (LEO) will set unprecedented performance in terms of connectivity and latency globally. By end of 2020 more than 500 LEO satellites were launched from roughly 200 at the end of 2019. The LEO mega-constellations proponents have announced more than 16,000 satellites in the next 3-6 years, potentially doubling the about 8,700 satellites that have been launched in the last 50 years. Starlink, promoted by Elon Musk, CEO of SpaceX is targeting service in North America and Canada in 2020, rapidly expanding to near global coverage of the populated world by 2021. A common objective of satcom proponents is to bring affordable high-speed access to every citizen and institution thanks to economy of scale in constructing and launching low mass LEO satellites. This paper starts introducing the evolving telecom needs for the ERTMS that is a de-facto global standard solution for train signaling in the world. The ERTMS connectivity requirements are evaluated looking to future years when GSM-R will have to be replaced by FRMCS whose high level architecture has been defined by the 3GPP Release 15. The FRMCS, as shown in Fig. 1 is connected to $3 \mathrm{GPP}$ radio access networks as cellular networks, non 3GPP radio access networks as the satcom, other than wirelines.

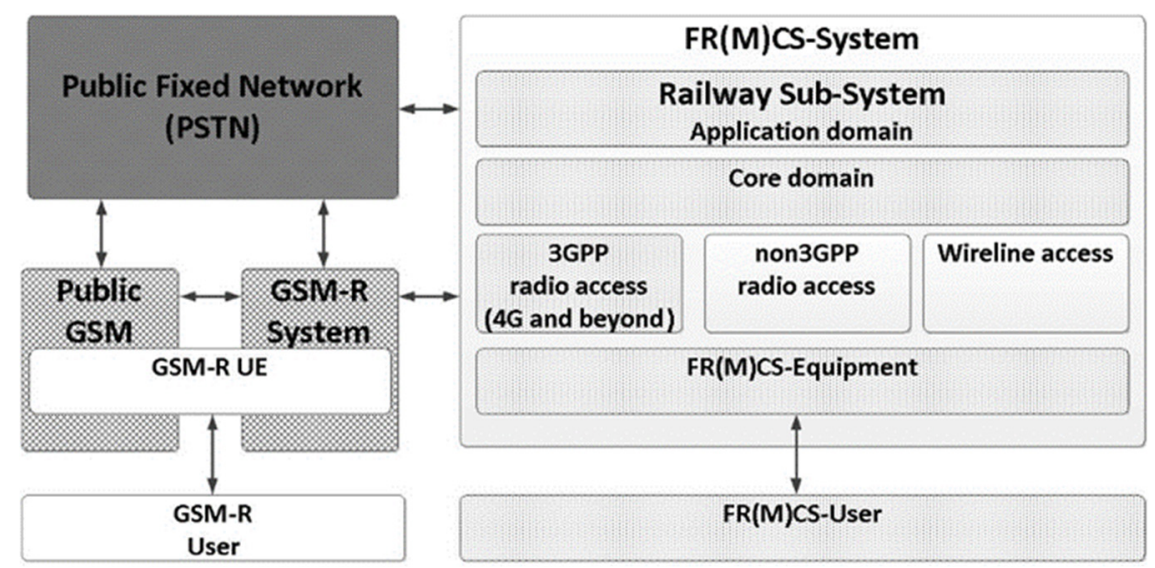

Figure 1: High level architecture of FRMCS. 
The new requirements are then analyzed assuming a multi-bearer platform processing a combination of cellular and satcom bearers. The end results in terms of potential cost savings are shown in parametric form after having identified some realistic operational scenarios.

\section{TRAFFIC CAPACITY EVOLUTION}

The starting point is the ERTMS deployment status as indicated in Fig. 2 which clearly shows the global footprint of the ERTMS at end 2019 [4], [5]. Europe holds about 51\% of tracksides and $69 \%$ of vehicles contracted. The globalization of the ERTMS is one of its strengths and to preserve this characteristic is important to look to different telecom networks to optimise the system according to regional constraints. For example in Australia, Hitachi Rail STS has deployed a TETRA network.

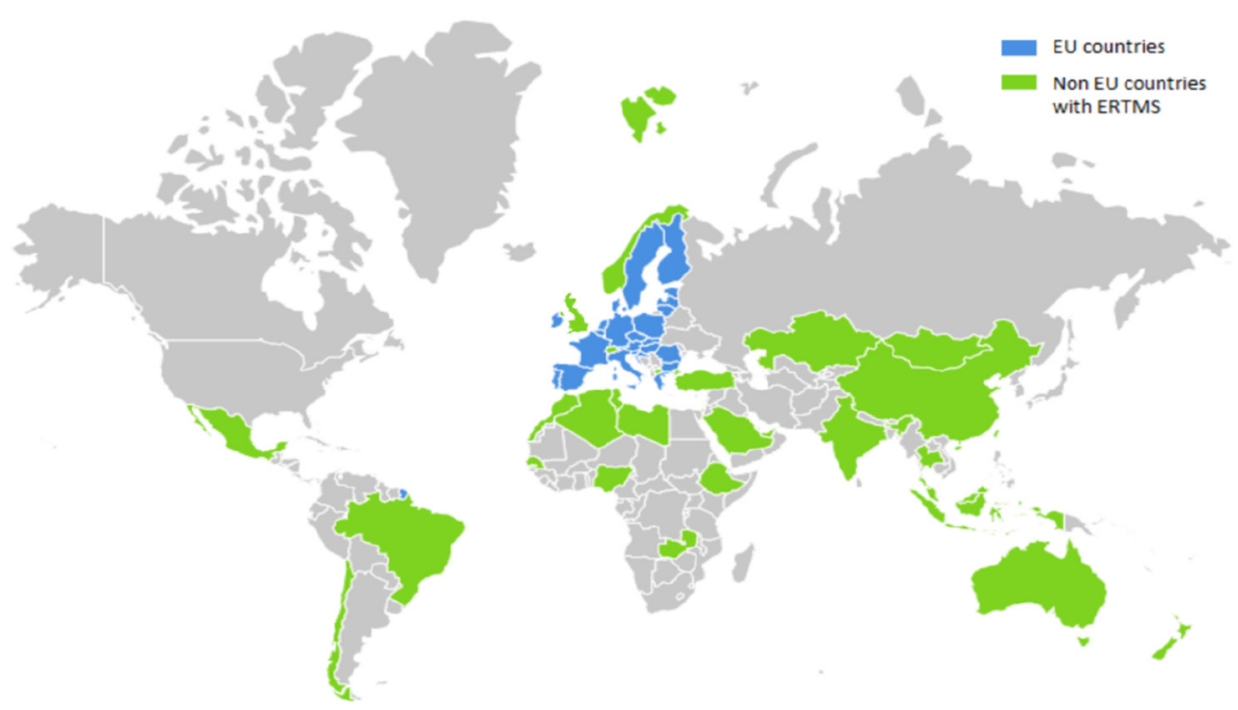

Figure 2: Status of ERTMS deployment/planned.

Looking in perspective, ERTMS will be evolving by adding new features which in turns increase the connectivity requirements. Here below there is a list of identified new applications that can be added to the ERTMS in a stepped manner starting from the basic services provided by the GSM-R and according to market needs [6], [7]:

- Current GSM-R critical applications: Voice (including emergency calls), ETCS L2;

- Train positioning;

- Automatic train operation (ATO) up to GoA2 (driver is still present in the cab);

- Remote control of engine;

- On-board intelligence and sensing (e.g. for ATO and object/person detection);

- Critical video (e.g. for remote control in shunting yards and in degraded mode);

- $\quad$ ETCS L3;

- ATO up to GoA4 (full automation, driver is not needed in the cab);

- Virtual coupling;

- Shunting voice;

- Other applications. 
Table 1: Target traffic needs for each area type.

\begin{tabular}{|l|c|c|c|}
\hline & $\begin{array}{c}\text { DN link traffic } \\
\text { per train }(\mathrm{Mb} / \mathrm{s})\end{array}$ & $\begin{array}{c}\text { UP link traffic per } \\
\text { train }(\mathrm{Mb} / \mathrm{s})\end{array}$ & $\begin{array}{c}\text { Number of } \\
\text { trains } / \mathrm{km}\end{array}$ \\
\cline { 1 - 2 } Low density & 4.38 & 7.42 & 0.33 \\
\cline { 1 - 1 } High density & $(0.42 \mathrm{w} / \mathrm{o}$ video $)$ & $(3.46 \mathrm{w} / \mathrm{o}$ video $)$ & 0.67 \\
\cline { 1 - 1 } High speed & & 0.5 \\
\hline
\end{tabular}

The foreseen aggregated traffic resulting from the above applications is indicated in Table 1 together with the number of trains/ $\mathrm{km}$ for three type of lines.

The overall connectivity for the whole European trains (EU-28) is estimated assuming that the pick instantaneous number of trains (PINT) is 26,000, excluding high speed trains, and applying the traffic scenarios of Table 1. Even though PINT varies from country to country according to their traffic patterns, the forecasts indicated here below are weighted to get an upper bound of the connectivity with the actual train traffic. The results indicate a range between $310 \mathrm{Gbit} / \mathrm{s}$ (upper level with video) and $110 \mathrm{Gbit} / \mathrm{s}$ (lower level w/o video):

(a) $11.8 \mathrm{Mbit} / \mathrm{s}$ per train (video included)=310 Gbit/s;

(a) 3.9 Mbit/s per train (video excluded)=100 Gbit/s.

To guarantee this connectivity with a dedicated telecom network, the actual GSM-R spectrum is not sufficient also because the GSM-R network has to be active during the migration to FRMCS. The hypothesis is to using the extended $1.4 \mathrm{MHz}$ allocated to GSM-R bringing the total spectrum to $2 \times 5.6 \mathrm{MHz}$ and reusing as much as possible the current radio network infrastructure. An additional spectrum of $10 \mathrm{MHz}$, possibly in the $1,900-1,910 \mathrm{MHz}$ frequency band is also necessary. Alternatively, this extra spectrum would not be needed at all or partially if the approach is to sharing public telecom networks with commercial mobile and satellite networks. In this scenario, railways would either become users of public networks embedded into the ERTMS delivery or operate as a mobile virtual network operator (MVNO) interfacing with the ERTMS solution providers.

Whilst cellular mobile operators have capacity in excess to offer to the railways, satcom operators will be capable to fulfil this potential demand once new high throughput GEO and LEO orbiting satellites will be available. In spite of higher cost, satcom is essential to guarantee the QoS since satcom connectivity is pre-emptible for a specific service/area all the time. Fig. 3 gives the whole satcom connectivity as function of the share of satcom connectivity respect to the total connectivity required to account for different operational scenarios. A $5-10 \%$ satcom connectivity share is typical for railways where at least three cellular networks ensure the coverage, while higher percentages apply to rural railways with poor cellular coverage. However, a trade off to optimise the satcom usage should be made for each specific case. Another interesting scenario is to use satcom as intelligent back-up of a terrestrial railways network. In that case the network/base stations may use less equipment for redundancies with less stringent and costly service level agreements because traffic is routed to satcom in case of failures on ground network equipment.

\section{REFERENCE TELECOM ARCHITECTURES}

Multi bearer architectures with satcom have been tested in Sardinia in 2015 [8] and are currently employed in Australia on the AutoHaul system [9]. In the first case, Hitachi Rail STS has tested both a public-only telecom scenario and a scenario where satcom acted as 


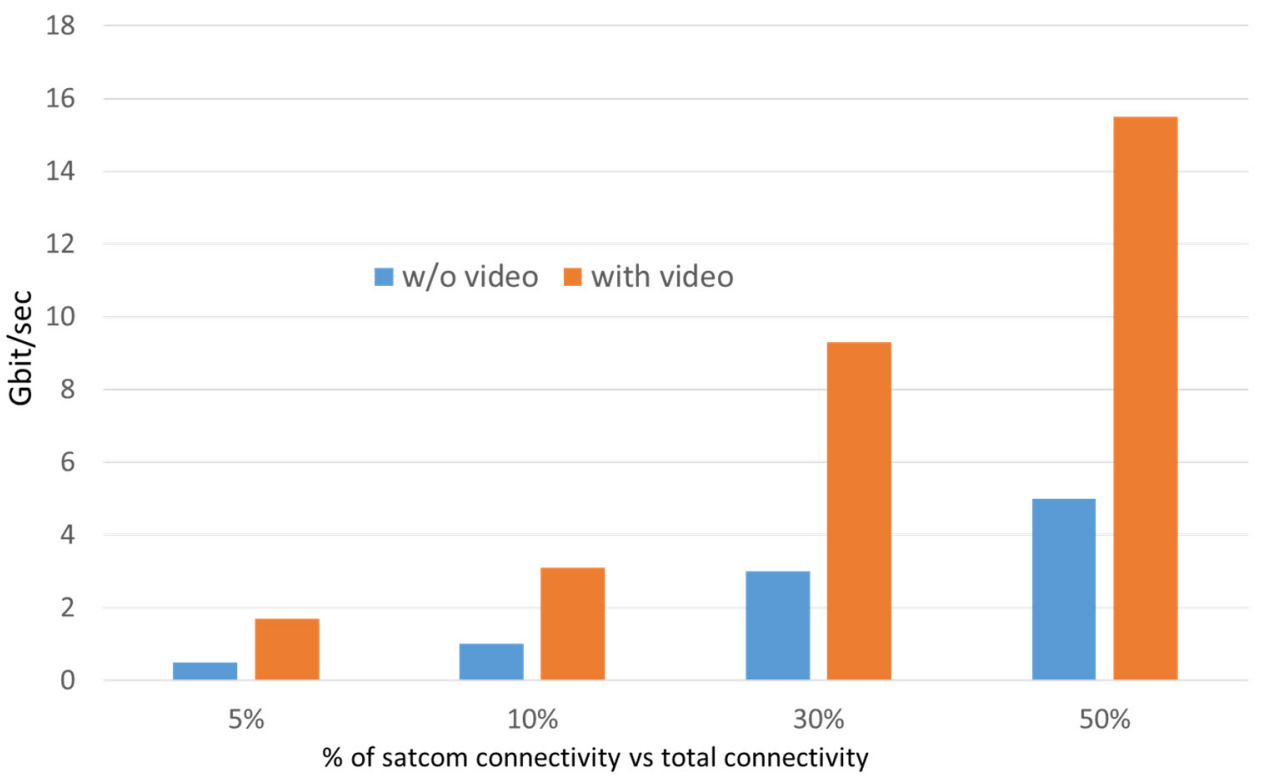

Figure 3: Satcom throughput versus different utilization scenarios.

back up to a TETRA network. The AutoHaul system uses the BGAN service of Inmarsat that is today a bench mark, having reached more than $500,000 \mathrm{~km}$ operating as the only telecom bearer for the Automatic Train Protection (ATP) and Automatic Train Operation (ATO) functions. Fig. 4 shows our roadmap, starting with the Sardinia test bed where we used the $2 \mathrm{G} / 3 \mathrm{G}$ cellular networks of three mobile operators in addition to the BGAN satcom and a TETRA network. The second step is based on field trials with a dedicated train running on selected lines in north-west Italy using 3G/4G cellular connectivity and two Satcom bearers (BGAN, Thuraya). This platform is part of the satellite-based ERTMS pilot line Novara Rho of RFI [10]. In this project a novel multi-link communication platform (MLCP) has been developed and tested. It is compliant with new European standard guaranteeing the backward compatibility with the GSM-R legacy networks and is ready for 5G and LEO satcom [11].

The last step entails the test of some of the new FRMCS applications over a $5 \mathrm{G}$ operational network. This activity is planned in the Radiolabs test bed at l'Aquila, one of the five cities in Italy with a $5 \mathrm{G}$ network to roll out pre-commercial services. These tests will be carried out in the urban and semi-urban environment using a vehicle equipped with $5 \mathrm{G}$ terminal [12]. The $5 \mathrm{G}$ test bed will be also used to evaluate the integration of satcom systems (including LEO) with terrestrial $5 \mathrm{G}$ network in order to prepare the ground for future terrestrial-satcom applications. In fact, differently from $4 \mathrm{G}$ networks the integration/interoperability of satellite systems in 5G terrestrial network has been explicitly considered and inserted in the 5G standardization roadmap. In particular, the main roles envisaged for satellite in the $5 \mathrm{G}$ networks are to provide:

- backhaul for the $5 \mathrm{G}$ access network;

- backup for the 5G network (useful in specific applications);

- differentiated/redundant communication paths (to be used with MP-TCP protocols). 


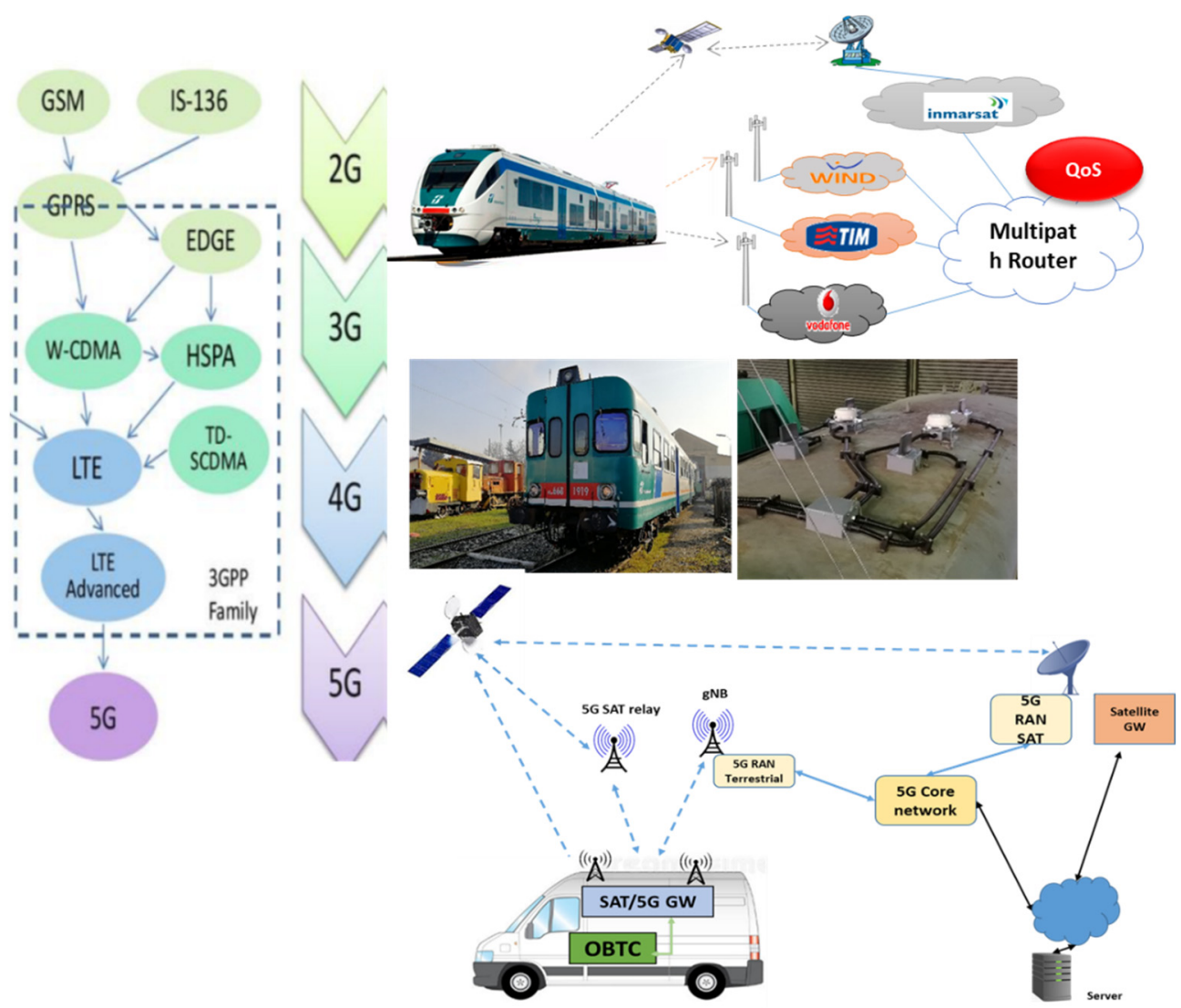

Figure 4: Phased approach to test public and satcom bearers.

The effort over the past 5 years and the growing consensus achieved so far are confirming the possibility to share public networks for ERTMS applications. The combination of cellular network with satcom, for prioritization and pre-emption, suggests a way to get a QoS for critical applications overcoming the difficulties (and costs) to imposing liabilities to MNO.

In the long term with the deployment of $5 \mathrm{G}$ networks this approach will become even more attractive using the network slicing capability to guarantee the QoS and satcom to extending the $5 \mathrm{G}$ footprint. Yet, resiliency and cyber security are important topics when using public access networks. In this respect a multi-bearer platform relying on physical separated networks is in principle more robust respect to a single network. Furthermore artificial intelligence techniques can be adopted to detect and mitigate possible interferences.

The concept of bearer independence has been selected by the Shift2Rail - X2Rail-1 project with the Adaptable Communication System (ACS) as the future railway communication infrastructure (Fig. 5). ACS provides an adaptable train-to-ground communications system usable for train control applications in all market segments, using traditional packet switching/IP technologies such as GPRS, EDGE, LTE, Satellite, Wi-Fi and other technologies. ACS is designed in accordance with the bearer independent principle (BIP) meaning that the ACS can interface with several and different communication bearers 


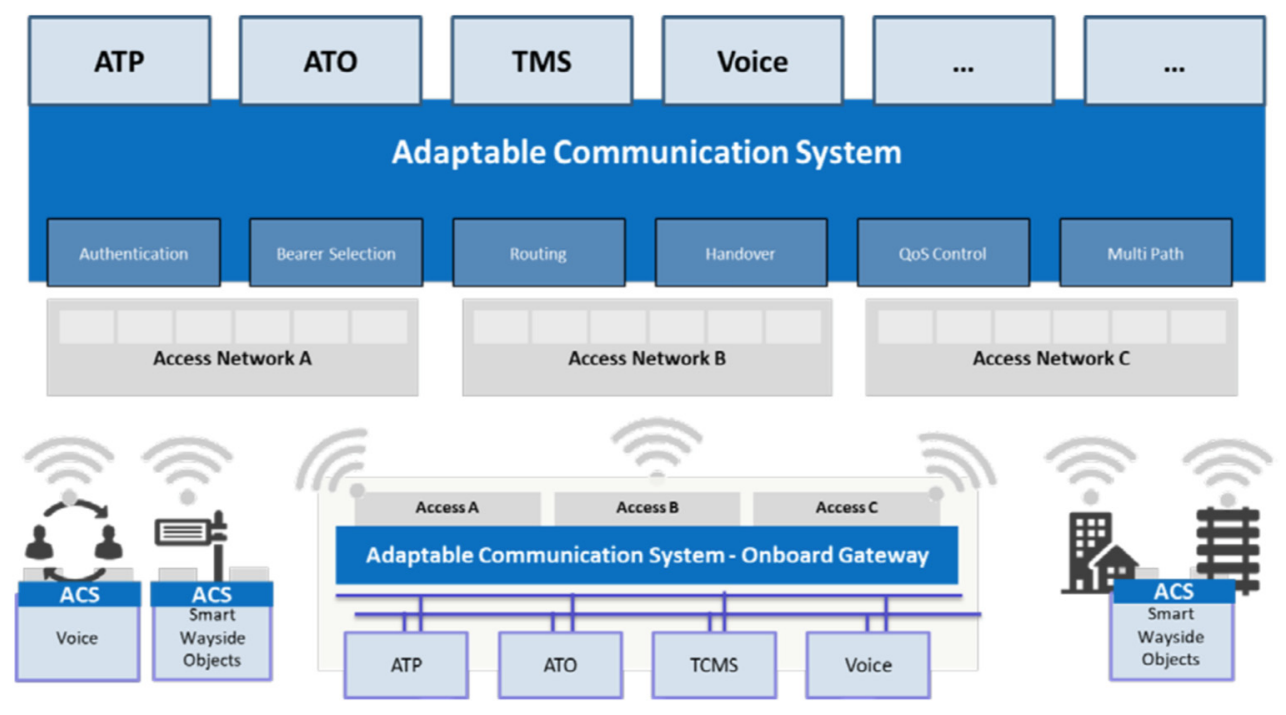

Figure 5: Adaptable Communication System (ACS).

(wired/wireless) to guarantee the end-to-end connectivity for railway applications. Interfacing of ACS with the bearers is made at IP level to fully implement the BIP concept. Communication of applications over the ACS can be provided over one or more IP-based bearers both alternative and/or traditional, public or private, without assuming that the bearer provides any capability beyond data transmission using the internet protocol (IP) [13].

Given the flexibility of ACS, is possible to design end2end connections by selecting proper bearers for the specific line and application. The economic benefits achievable with public bearers have been analyzed in a number of use cases by comparing the total cost of operations over 10 years with the costs of a dedicated telecom network. The business models are different: with public networks, the costs for a turnkey telecom service are proportional to the number of circulating trains; instead, for a dedicated network such as the GSM-R the costs including the installation of the network, the maintenance and operation depend on the length of the railways line. To help understanding the advantages of using public networks a mix between number of trains and railway lengths has been analyzed (Fig. 6). The benchmark is the cost of the GSM-R network, estimating an average value between a green field implementation and the upgrade of an existing network. Furthermore, it has been assumed that the end2end connectivity is achieved by using satcom for $20 \%$ and cellular networks for $80 \%$ of the train trip. The final results which must be considered preliminary due to the starting hypothesis, indicate a trend of significant cost savings with public networks in all the analyzed scenarios. On that basis a more accurate analysis should be done for each line by taking into account its characteristics.

A side-benefit when using public telecom networks is the reduction of $\mathrm{CO}_{2}$ emissions. In fact, the electricity to feed $\mathrm{H} 24$ the base stations of a dedicated network is responsible for circa 60 to $100 \mathrm{~kg}$ of $\mathrm{CO}_{2}$ emissions per day per $\mathrm{km}$. Extrapolating this value to $30,000 \mathrm{~km}$ of regional lines in Europe, a potential saving of about 1.8-3 million $\mathrm{kg}$ of $\mathrm{CO}_{2}$ emissions per day seems possible with public networks, assuming that $50 \%$ of the electricity is generated by renewable means. 
3000

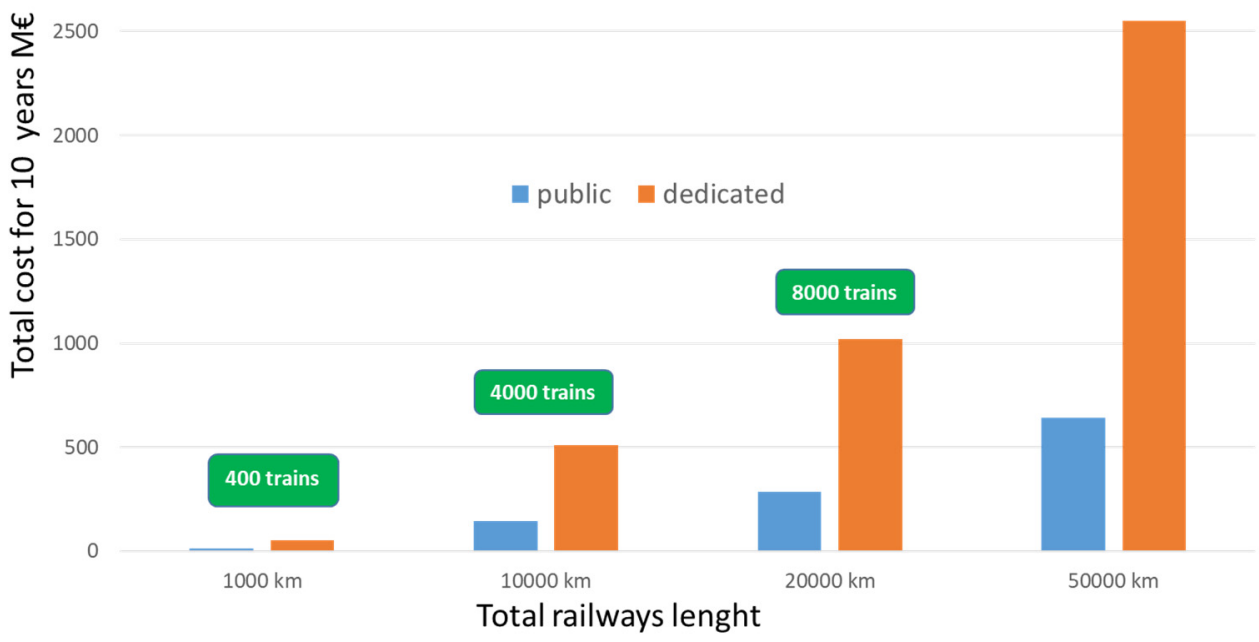

Figure 6: Estimated total cost of telecoms over 10 years.

\section{FUTURE DEVELOPMENTS}

Under TEN-T Regulation the European Member States are in principle obliged to deploy ERTMS on all the Core Network railways by 2030 (about 65,000 km) and 2050 for the Comprehensive networks (about 122,000 km). Yet, the fleet to be equipped by 2030 is estimated in the range of 27,500 to 38500 vehicles, compared to some 3,600 vehicles already equipped in Europe. In the frame of the ERTMS deployment, RFI has announced a plan in Italy to install ERTMS all over their lines by decommissioning the class B systems, thus becoming a full user of the ERTMS [1].

One of the great challenges ever faced by the rail sector is to satisfy the demand for communication during the migration from GSM-R to FRMCS and fulfilling at the same time an increase of train connectivity during the next years. A stepping stone is the evolution from circuit switched based communication systems towards IP-based communications, opening the way to a multitude of telecom bearers, instead of just one underlining technology as was the case of GSM-R.

To satisfy the increase of throughput, European Commission is evaluating an interim $1.4 \mathrm{MHz}$ spectrum adjacent to the GSM-R band as well as the $1,900-1,910 \mathrm{MHz}$. Both solutions impose constraints since the new $900 \mathrm{MHz}$ band - adjacent to those used by legacy mobile network operators - may need power restrictions to avoid adjacent channel interference, while the $1,900 \mathrm{MHz}$ band will imply a higher number of base stations along the railways, thus increasing the costs.

A way to limit additional spectrum is to rely on public networks as described in this paper having recognised that in the railway environment, spectrum resources dedicated exclusively to railways may be insufficient, not available, difficult to coordinate with other users and perhaps not efficiently used since base stations are idle for most of the time being active just when train is within their footprint. Under these circumstances communication services offered by commercial mobile and satcom network operators should be considered strategic resources to alleviate these problems. 
Sharing public networks would allow also to minimize on a national basis additional spectrum needs. From a technical point of view, cellular and satcom networks form a complementary mutually-sustainable bundle ensuring at the same time a whole coverage and QoS, as demonstrated with field tests in Italy. Resilience and availability, two peculiar characteristics can be achieved by the on-board multi-bearer processor on top of the standard bearer quality provided by the mobile network operators (MNO).

This aspect would entail further developments exploiting for example artificial intelligence with extensive field tests for training algorithms to optimize bearer selection along the railways lines. taking advantage of trains travelling frequently on the same lines.

Wi-Fi can also be used as additional bearer to off load the amount of data carried on cellular networks in proximity of stations where peak of capacity is generated by the simultaneous presence of many trains and satcom coverage may not be always ensured. In perspective, the development of $5 \mathrm{G}$ is set to revolutionize mobile services for railways, introducing in particular the network-slicing feature that allows to create a virtual network with guaranteed QoS. Network slicing - a breakthrough of $5 \mathrm{G}$ - is ideal to guarantee the railways specific connectivity needs and to exploiting also new train to train communications.

To meet the requirements of railways applications, $5 \mathrm{G}$ networks must ensure a coverage also outside main cities and high density areas where 5G networks are deployed at the beginning. In this respect, satcom are already evaluated at ETSI to foster the roll out of 5G service in unserved areas that cannot be covered by terrestrial $5 \mathrm{G}$ network (isolated/remote areas) and underserved areas (e.g. suburban/rural areas). In other words to upgrade the performance of limited 5G terrestrial networks in cost effective manner [16].

The mobile ecosystem for railways is evolving out of the traditional GSM-R supplier model and, other services for the passengers like web browsing, streaming, video surveillance, are boosting demand of connectivity in the Gbit range for the whole train. Such "backbone connectivity" on board trains travelling up to $300 \mathrm{~km} / \mathrm{h}$ represents a real challenge also for the faster cell handovers due to a smaller cell size of the mobile broadband networks. The new paradigm of bearer independence backed by proper cybersecurity means is opening a wave of innovation in the railways sector. Similarly to aviation where a new satellite-based air-ground communication system (IRIS) is being developed for Air Traffic Management. (ATM). Actually pilots communicate with Air Traffic Control (ATC) by voice, over radio frequency channels suffering congestion, and with the projected doubling of aircraft in Europe's airspace by 2035 a shortage of communication may impact on air traffic, causing bottlenecks at airports. A study to evaluate the IRIS system for rail applications has been recently completed for ESA [16].

Synergies should be explored also with the automotive sector - by far the biggest user of vehicle to vehicle (V2V) and vehicle to infrastructure (V2I) technologies fulfilling safety requirements for connected and autonomous vehicles. These technologies are starting to be deployed along the roads to communicate with vehicles and in perspective could be shared with the railways being railways and roads generally close to each other. Yet satcom technologies for railways, already evaluated by ERA, are an important topic for ESA in supporting the introduction of the FRMCS in cooperation with ERA. The underlining objective is to foster the evolution of ERTMS with a building block architecture modular, upgradable and always connected [17], [18].

Whether railways communications will move gradually, endorsing new technologies will depend also by the mobile and satcom operators. Railways communication requirements have been predicted relying on large consensus for a steady migration to the FRMCS. It is almost certain that ERTMS competitivity will increase upon its ability to manage efficient, robust and high speed communication channels. Time is different from the early 1990s when 
GSM-R has been selected and since then has contributed to the success of the ERTMS in the world. At that time, this choice was driven by a stepping innovation on mobile communications brought by the GSM, having had the merit to set a standard for ensuring the interoperability of ERTMS. These criteria still apply to the FRMCS, the successor of GSM-R, but with a technology-independent vision and more attention to the economical sustainability in stark contrast to past years. Interoperability will not be an issue with standard public mobile networks while satcom bearers would be selected to ensure as minimum a European coverage.

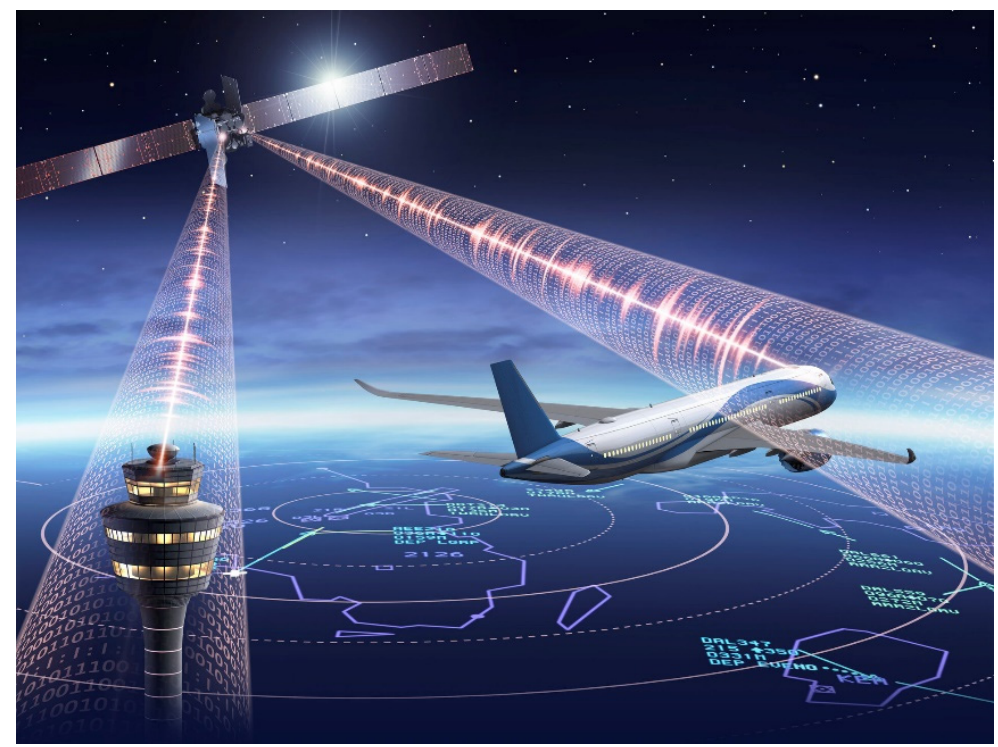

Figure 7: IRIS for air traffic control - ESA.

\section{ACKNOWLEDGEMENT}

Special gratitude is due to RFI and Trenitalia that are supporting the field tests within the projects co-funded by ASI (Agenzia Spaziale Italiana) through ESA and to Radiolabs for the use of the $5 \mathrm{G}$ test bed in l'Aquila.

\section{REFERENCES}

[1] European Court of Auditors, A single European rail traffic management system: Will the political choice ever become reality? Special report, 2017.

[2] FRMCS, UIC report. https://uic.org/rail-system/frmcs/.

[3] Shift2Rail-ACS, https://projects.shift2rail.org/s2r_ip2_n.aspx?p=X2RAIL-1.

[4] UNIFE, Report on ERTMS. www.unife.org/publication-press/publications/171annual-report-2019-executive-summary.html.

[5] ERTMS, First Work Plan of the European Coordinator Matthias Ruete, May 2020. https://ec.europa.eu/transport/sites/transport/files/work_plan_ertms_2020.pdf.

[6] CEPT, Report 74 on spectrum needs.

[7] FW-ATwG 1903 v2.2.0/FM56(18)065: FRMCS Traffic Analysis.

[8] https://business.esa.int/projects/3insat.

[9] www.riotinto.com/news/releases/AutoHaul-successfully-deployed. 
[10] ESA Space4Rail, Perspective of space utilisation in the Italian Railways, 13 Nov. 2019, Massimiliano Ciaffi, RFI Fabrizio Memmi, Trenitalia.

[11] https://artes.esa.int/projects/sat4train.

[12] www.radiolabs.it/en/radiolabs-unveils-the-initiative-emerge/.

[13] X2R1-WP3-D3.3-Communication system, Specification and technology guideline.

[14] RFI ERTMS Implementation plan in Italy: A system technological strategy, CCRCC2019 ERTMS Conference, Fabio Senesi.

[15] 3rd Generation Partnership Project (3GPP TM), Study on New Radio (NR) to support non-terrestrial networks, Release 15.

[16] ESA, IRIS for railway communications, Final Report, 2019.

[17] Final Report, ERA study on feasibility of SATCOM for railway communication.

[18] The ERA view on the future rail system, CCRCC 2019, Valenciennes Josef Doppelbauer, Executive Director. 